\title{
A Framework for Modelling Mobile Radio Access Networks for Intelligent Fault Management
}

\author{
Marwan Awad \\ Sudanese Knowledge Society \\ Khartoum, Sudan \\ email: m.adam@sksociety.org \\ dot.energy, Sudan and UK \\ Email: m.awad@dotenergyconsulting.com
}

\author{
Hassan Hamdoun \\ Research Fellow, School of Natural and Computing \\ University of Aberdeen, Kings' College, Aberdeen, UK \\ email: hassan.hamdoun@abdn.ac.uk \\ dot.energy, Sudan and UK \\ Email: h.hamdoun@dotenergyconsulting.com
}

\begin{abstract}
The increased level of heterogeneity among the network components of current mobile Radio Access Networks (RANs), has meant that conventional network troubleshooting techniques are no longer sustainable; impacting negatively on customer experience and Quality of Service (QoS). Intelligent Fault Management (IFM) is a promising approach that utilizes the power of data mining in addressing emerging and future challenges in RAN network troubleshooting and performance management. This paper provides a comprehensive integrated RAN modelling framework in order to reflect protocol, topology, service inventory, and correlations between Alarms in a typical Mobile Radio Access Network (RAN). It proposes and presents results from a RAN model leading towards an effective Network Management System (NMS) datasets that could be analysed to discover alarm rules, associations and core drivers using different data mining techniques, graph theory measures and data benchmarking methodologies.
\end{abstract}

Index Terms-Radio Access Network (RAN), Intelligent Fault Management, Data Mining, Network Graph and RAN Model

\section{INTRODUCTION}

Radio Access Network (RAN) in the Mobile Service Provider (MSP) network is one of the most important layers. The RAN provides a direct interface with subscribers using many resources and hence is the MSP network accessibility layer. The healthiness of this layer has a direct impact on customer experience and on the business of the MSP. Fault Management (FM) concerns with the healing process of the mobile network, among other Network management

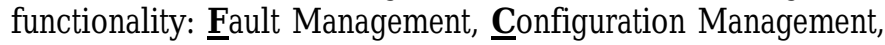
Accounting Management, Performance Management, and Security Management (referred to as FCAPS), FM is the most human time and expertise demanding. The traditional approach utilizes operation staff experience and intuition to troubleshoot, localize and correct faults. Today the complexity and heterogeneity of the access layer has meant that MSPs are faced with vast amount of reported alarms that make manual handling unreliable. Another challenge relates to the required knowledge needed to troubleshoot/solve the technical fault, which, is usually quite tacit in nature and is built from engineers' long experience. Moreover, the knowledge remains personal and usually gets lost by job transfer. Hence, addressing the issue of knowledge acquisition in the FM domain is a challenging research topic.
In the quest for autonomous network operation and service provisioning, data mining, machine learning, and artificial intelligence approaches have been introduced within the context of self-healing for automating FM functionality. For example, alarm correlation is discussed in [1] with focus on reducing alarms floods and increasing the semantic content of the faults presented to the operator. Mining patterns in alarms databases is one of useful discovery methods used, however, efforts in mining alarm associations has not been able to reflect the real challenge facing telecommunication networks. This was mainly due to that the schemes employed are guided by flat market basket analysis resulting in multidimensional problem formulation [2]. Temporal relationships in mining alarms are studied in [3] with focus on the topographical proximity and topology when mining sequential pattern association. The current approaches, though, fall short in reflecting the topological and temporal dimensions when modeling the RAN.

RAN Alarms are usually reported as a message notification reflecting the local status of the reporting device, and then categorical classes are created at the Operational Support Subsystem (OSS) level. The interpretation of the alarm is often human-oriented process spanning narrow time window and making long term analysis or automatic online extraction of alarm semantic content difficult.

Assuming the challenge of developing models that encapsulate the topology relationships and network/services dependencies with outcomes measured at the business layer, semantic content of alarms becomes a key methodology to adopt. In order to enrich and integrate the semantic contents of alarms within a global view in the OSS system, Object Oriented Programming (OOP) can be used. OOP enables modelling static abstraction of the RAN functionality and architecture. OOP also facilitates handling dynamic network events (Alarms list) using the static information enriched model (Managed Object class) in a flexible manner providing a robust fault diagnosis and interpretation. Using this approach, the Radio site will represent the basic building block containing all the resources that have a direct impact on the service area while facilitating the modeling of other RAN resources such as Base Station Controller (BSC), Transcoder Controller (TRC), and backhauling links.

This paper deals with modeling of RAN in order to reflect temporal, topological resources/service dependency, business 
impact and integrated semantic content of the alarms, with the aim of aiding the process of knowledge discovery and robust alarm correlation in alarm databases. The outline of the paper is organized as follows: Section II presents the related work to Fault Management. Section III describes the modeling methodology. Section VI describes how the network topology and graph representation model used. The Topological features and performance measures are discussed in Section V. The Numerical results are presented in Section I. . Section II draws the conclusions and outlines the future work.

\section{RELATED WORK}

To overcome the network heterogeneity problem, two approaches were adopted: i) standardizing the management network and ii) raising the abstraction of the representation of resources. Integrating Network Management Systems (INMS) is an attempt to bring order to the chaos. INMSs must be built around commonly accepted standards so that products from multiple vendors can coexist and interwork [1,4]. The International Telecommunications Union (ITU) has classified network management into five different functions, which are called FCAPS in short. The TMN also define the aggregation of responsibilities into very loosely coupled layers: Network Element Layer, Elements Manager Layer, Network Management Layer (all are Resource oriented), Service Management Layer, and Business Layer. Each of the FCAPS functions focuses only on one specific function across these layers without much interaction with other functions.

The first subset of the research work in IFM focuses on alarm correlation as a diagnostic tool for alarm number reduction. Increasing the semantic content of the alarm via alarm correlation is presented in [4]. Different Coherent potential proximities for alarm correlations are considered. Behavioural Proximity (BP), with the main objective of clustering alarms by behaviour of events is studied in [5]. The event Duration matching (EDM) algorithm is proposed an implemented in [5], resulting in a set of alarm clusters. Further research, building on topological proximity (TP), has proposed structural proximity approach [18]. Approaches relying on system description-based diagnosis are presented in [7] where axioms characterizing the behaviour of various types of systems components e.g. base stations transceiver of a given vendor are detailed. The topology is then modelled separately building on several other information and facts about the system. Given the topology and alarm correlations, efforts were focused on finding potential sequential alarm patterns with dependency and causality characteristics. Not only finding those patterns will reduce the number of alarms, but also sequential alarm data mining can produce associated patterns resulting in increase in the semantic content of alarm correlation. Note, that still temporal proximity [8], behavioural proximity and topographical proximity [9] are used in order to increase accuracy of semantic content found in those alarms.

The second subset of the research work in IFM focuses on aggregation of network layers to match business aspects, mainly Resources Layers (NE, NEM, and NM Layers), Service Management Layer and Business Management Layer [10].
Dependencies between layer services at the BSC are modelled as a directed acyclic graph (DAG), where each node represents a sub-network, and an edge indicates functional dependence among sub-networks. Service dependency graphs with nodes as Managed Objects (MOs) and edges as service relationships are presented in [11]. Policy-based service management graphs with Service Level Agreement (SLA) guarantee are presented in [5].

The final subset of research work in IFM, is built on knowledge based (KB) extraction. Alarm correlation uses rule based expert system, model based system codebooks and knowledge discovery from databases (KDD) for the diagnosis, localization and mining of sequential alarm patterns. New approaches using different types of knowledge are utilized in IFM in order to model the alarm propagation behaviour and dependency [2, 12, and 13], e.g. data mined for detecting sequential alarms patterns [7] or Bayesian Belief Networks [5]. Such modelling approaches have succeeded in accounting for process knowledge, expert knowledge acquisition from staff, domain ontology and case-based reasoning (CBR) [14, 15]. All subsets of research work on IFM have themes in common making them complimentary and facilitating for our integrated modelling approach. Hence, the approach employed in this paper relies on modelling the RAN taking into account all integration aspects that supports diverse aspects of fault management process. In particular the OOM in order to increase the level of system abstraction is implemented. Such approach will ultimately facilitate RAN resources and architecture abstraction within a multivendor NMS context.

\section{METHODOLOGY}

The hardware architecture in the Base Station Transceiver (BTS) is not directly visible from the BSC, so vendors develop a model for the BTS that is used both in the BSC and the BTS. This model is a logical representation of the hardware and software functionality, describing the BTS in a functionaloriented way. The model is presented as a set of MOs where a MO does not necessarily have a one-to-one relation with a physical unit in the BTS. The MO comprises both hardware and software, or software only. Many different types of logical models in the BTS have been developed to adapt with several different hardware implementations of BTS.

The approach described in this paper is generic illustration of how the Managed Object Model (MOM) (with MO as smallest entity) of the BTS can be extracted from the alarm message and mapped in MOM tree topology. This paper implements a set of $\mathrm{MO}$ aware parsing tools that convert plain alarms text (an example of which is shown in Fig.1.) into a database ready for graph analysis and data mining. The following subsections describe relevant resources and components of the system.

A) Radio Site

The radio site represents the basic access point that serves a certain geographical area. It consists of many network's and facility's resources such as BTS, Node-B and Node-E (Radio Resources), Microwave and ADM (Transport resources), Generators Set, Rectifier and Battery Backup (Power 
Resources) and Climate Control System such as Air/Conditioner (A/C). Traditionally, the reported alarms have not been exploited to produce explicit site representation despite that the alarm content text included some sort of reference that can directly or indirectly pinpoint to the radio site. Out model utilizes two paradigms supporting each other: relational database to support traditional alarm list and OM to encapsulate the content, topology and dependency relations of the radio sites' equipment and their behaviours.We also developed a parsing tool that converts alarm text and saves it into the database. The model is able to correlate each triggered alarm with a certain site. The radio sites, in turn, have direct relation with the affected object or with the service inventory that affects the site coverage. The philosophy behind this model enables each event at the BSS level to be linked directly to a radio site. This site usually shows a topology alarms propagation because of RAN's backhauling structure, power and environmental dependency which also affects other resources in other sites through a generic method (mappAlarm).We manipulate the process of mapping alarms from the Active Alarm list to the affected site. For example, applying mapAlarm on the following fragment of Alarm list will assign the external fault to the radio site 'WoodShop'. The Alarm is reported from TG-35(Ericsson Company's naming convention).

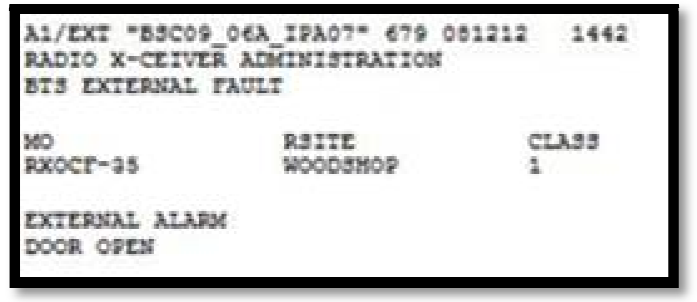

Fig. 1 Alarm Fragment

\section{B) Radio Resources}

The radio site is usually comprised of $3 \mathrm{G}$ and $2 \mathrm{G}$ base stations (Node-B and BTS respectively). Both Node-B and BTS are directly related to MOM of the BSC and Radio Network Controller (RNC) with small modification to object naming to reflect the generic abstraction of similar resources. This paper deals only with $2 \mathrm{G}$ components. The BTS class which represents the TG Model uses the same BSC MOM with additional helpful information. The BTS recourses with its geographical mapped cells are the most important parts of RAN, and which we use in this paper. A typical site consists of 3 TGs and one Node-B so that it provides both $2 \mathrm{G}$ and $3 \mathrm{G}$ services. It is clear that the site encloses diverse set of resources which represent different systems. The radio resources are directly associated to the service layer where the cells usually represent the logical aspect of geographical mapping and dedication of resources to serve a certain geographical area. Beside cell representation, MO also relates to basic radio functionality both as software or hardware which needs to be represented and aggregated as service resources. Radio resources are modeled as TRU, TX, RX as basic functionality for Radio Frequency (RF) and some of Baseband basic functionality while synchronization, traffic routing, and BTS transport related functionality as well as Operation and Maintenance (O\&M) and control functions are modeled using Main Processing Unit (MPUs).

C) Transceiver Unit (TRU)

In GSM, the Transceiver Unit (TRU) is the main radio resource and all Trans/Receive functionality are modeled using this object. The object encompasses the TX object, RX object and basic traffic unit named a timeslot (TS). Each single TRU can handle certain maximum Erlang traffic units, and any malfunction related to core TRU functionality will affect the service and hence these impacts need to be estimated. Alarms affecting these resources must be studied carefully to assess their impact, probability and serviceability. There are two important mappings that are necessary for proper TRU modeling: First TRU as physical hardware and software resource. In this mapping many TRUs (up to 4 or even more) may be associated with one Hardware unit and hence the hardware is linked to logical representation of their basic functionalities. This mapping also allows resource representation, failure rate, Mean Mean Time to Repair (MTTR), Mean Time between Faults (MTBF) and failure type to be extracted from alarms text. The second mapping occurs when several TRUs are attached to a cell in and provide sufficient resources for serving certain geographical area.

D) Service and Business Revenue

The healthiness of network is related to the resources that provide the service with critical quality measures in the dimensions of (Accessibility, retainability and service integrity). Also resources malfunction is correlated with outage and availability, and hence these measures can be linked directly to revenue and customer experience. Using the same argument, data services can be related to the capability of resource in provisioning data stream with the measures of accessibility, latency, volume and integrity. Revenue Loss series for a cell (and site) can be estimated for a certain time point in a 24 hour traffic profile from the time the system reports theErlang values occupied each hour. A good estimation can be obtained when this is modeled between the revenue expected in that time point and the effect of offloading traffic to the neighbor cells. The business impact of the resource malfunction will be estimated accordingly; such direct mapping between physical resources, service, and business will enhance fault management significantly.

E) Transport Model

Since transport resources depend highly on the topology structure, RAN modeling should reflect this structure in order to facilitate finding the topology with the alarms root cause, alarms correlation or to assess service impact. Topology attributes such as parent, child, and path are very important. The suitable data structure to represent such node is a tree.

1) Hop

The backhaul network usually uses microwave transmission for implementing different technologies (E1, IP, MPLS, etc). In a typical RAN, the backhaul microwave link connects two sites, the physical channel and environment of this link is called a hop. Many network faults related to the transport network have 
direct relation to the engineering and environment of the hop (e.g. configured frequency, frequency deviation, TX power, Line of Sight (LOS), parameters, etc). The hop represents the transport links and is the sum of all individual connected sites routed through the link.

\section{2) Link}

The link refers to the basic building block of transport capacity terminated at the site. Conventional GSM link is the E1 (or T1), which implement the GSM's A-bis interface. The A-bis interface has a route from the BSC to each site; this route is affected by transport and power problems from all sites the route passes by.

3) Route

A route is the path between the site and the BSC and includes all sites (vertex in the graph) that the route passes to reach the BSC. Route information can be saved in an adjacency Matrix using the edge (hop) description.

\section{NETWORK TOPOLOGY AND GRAPH REPRESENTATION}

Radio site in the RAN can be presented as vertex, while the controller BSC or RNC act as the rootvertex. Alarm events occur and each alarm can be assigned directly to a vertex with its topological properties yielding some network and service status consequences. The microwave hop or any transmission medium that transports traffic between two sites will make an edge link between these two vertexes (sites). For each edge $\boldsymbol{e}_{i j} \in \boldsymbol{E}$ there are a set of A-bis paths (at least one) that passes it.

Given a RAN network with $\boldsymbol{n}$ sites (including the BSC or RNC), $\boldsymbol{E}$ backhauling edges, $\boldsymbol{A}$ alarm sets, $\boldsymbol{F}$ topological features associated with each vertex in $\boldsymbol{V}$, such network represent an attributed directed graph $\mathbf{G}=(\boldsymbol{V}, \boldsymbol{E}, \boldsymbol{A}, \boldsymbol{F})$. With the aid of microscopic and macroscopic topological properties or features suggested in [1], MIT network analysis toolbox [16], a set of topological features is selected and computed. By introducing topological representation and features the effect of how the network is connected will be considered in addressing FM analysis since this will encode potential vertices and edges properties.

Graph Topology is introduced in this research in order to reveal hidden topological properties due to transmission connectivity and MO hierarchies. Extracting such properties require executing some algorithms on the graph and hence raise the question of how we can represent the RAN network using graph tools. In graph network formulation we utilized the main common representations such as i) edge list: an $\boldsymbol{m}$ edges ordered pairs $\left(a_{i}, b_{i}\right)$ such that $e_{i}=\left(a_{i}, b_{i}\right)$. An extended edge list: contains an additional vertex coordinates [6], ii) Adjacency list: is a set of $\boldsymbol{n}$ lists $\left(A_{1}, \ldots A_{n}\right)$ where $\mathrm{A}_{\mathrm{i}}$ contains all vertices $\mathrm{j}$ for graph $\mathbf{G}$ such that it contains an edge $(\mathrm{i}, \mathrm{j})$ [16]. iii) Adjacency matrix: an $\boldsymbol{n x n}$ matrix A, such that $A(i, j)=1$ if $i$ is connected to $j$ and $A(i, j)=0$ otherwise. In this paper the RAN model is constructed using an edge list with latitude and longitude values that is converted to an adjacency matrix.

\section{TOPOLOGICAL FEATURES AND PERFORMANCE MEASURES}

This section reviews several metrics and their capability to describe certain aspects of our RAN model.

\section{A) Degree}

The degree of the node is the number of links adjacent to the node [3]. The total degree is the sum of all in and out-degree. For a given vertex $\mathrm{v}$ it can be written as:

$$
\operatorname{deg}(v)=|\{u \in V,:\{u, v\} \in E\}|(1)
$$

In the mobile telecommunication context this reflects the importance of the vertex (Radio sites) in backhauling connectivity. The higher the degree, the more impact a site has upon a transport or outage fault.

\section{B) Cluster Coefficient}

Cluster coefficient evaluates the connectivity of the neighbours of $\mathrm{v}$ and hence its local density. It is the extent to which the vertexes in the graphs tend to cluster together.

$$
\operatorname{CLUST}(v)=\frac{2|\{\{\mathrm{u}, \mathrm{v}\} \in \mathrm{E}:\{\mathrm{u}, \mathrm{v}\} \in \mathrm{E} \wedge\{\mathrm{v}, \mathrm{w}\} \in \mathrm{E}\}|}{\operatorname{deg}(\mathrm{v})(\operatorname{deg}(\mathrm{v})-1)}
$$

\section{C) Closeness Centrality}

Node centrality refers to the place of node in the network. Centrality measures can be performed on links or on paths [17, 18]. Closeness measure the closeness to all vertices, CLOSE(v) is the inverse of average distance between vand all other vertices that are reachable from $v$, the distance between two vertices is defined as the number of edges of the shortest path between them .

$$
\operatorname{CLOSE}(\mathrm{v})=\frac{\mathrm{n}}{\sum_{\mathrm{u} \in \mathrm{V} \mid \text { shortest_path(u,v) } \mid}}
$$

\section{D) Betweeness Centrality}

The betweeness centrality BETW(v) of a vertexv is equal to the number of times the vertex appears in shortest path in the graph. Since node betweeness is proportional to coincident paths that go through a node, it directly reflects how the transport topology in telecommunication networks depends on those vertexes (sites). The higher the betweeness centrality, the higher the impact of the vertex on the network.

$$
\operatorname{BETW}(\mathrm{v})=\sum_{\mathrm{u} \in \mathrm{V}} \|_{\text {shortest_path(u,v) }}(\mathrm{v})
$$

Betweeness can be measures for a node (vertex) or a link(edge). Edge betweeness is proportional to number of shortest path going through an edge as detailed in [5]. It directly reflects the importance of the edge. Refereeing to RAN network representation, this represents the rank of the link risk and its impact on the transport network.

\section{E) Eignvector Centrality}

Eignvector centrality is the eignvector that corresponds to the largest eigenvalue of the adjacency matrix, i.e. the $i^{\text {th }}$ 
component of this vector give the centrality score of the $i^{\text {th }}$ node. Whereas, the vertex Eign-centricity (of node i) is defined as the maximum distance to any other node.

\section{F) Managed object Topology}

Resources are hosted in radio sites has a MOM built in a tree like topology. Each managed object related to an alarm contains directly and indirectly this topological information. This can be extracted from the MO in $2 \mathrm{G}$ systems or from Full Distinguished Naming (FDN) in 3G systems. The topological information exploited from the alarm text is used to update the information in the MO tree. In GSM, the topological information is encoded in the MO e.g. RXOTRX-122-5 and RXOTRX-122-6 refer to two MO TRXs belonging to the same radio base station and RXOTS-122-6-4 refers to TS associated with TRX no 6 in Transceiver Group TG 122. Here every MO represents a node while the edge represents the parent child relation as well as system events.

\section{G) Performance Measures}

\section{1) Mean Time Between Events not Failure (MTBE)}

One of the major reliability KPI is MTBF which measures the reliability of devices or the system. In a RAN, the reported alarms are symptoms rather than failures. Accurate modeling of managed object behaviors and event probabilities is needed. Instead of deriving MTBF, which model the equipment reliability, in this paper we propose the use of Mean Time between Event (MTBE) in order to build a model about system functionality rather than system equipment reliability. The MTBE will measure the different aspects behind events from system functionality to system operation and engineering. It reflects the engineer's capability to optimize system operation and performance as well as system capability and behavior.

Event (Alarm) occurs due to system malfunction, operator's intervention and system configuration. From event statistics, a model that describe the dependency between events, system functionality reliability and operation performance capability can be built. MTBE and Mean Time to repair (MTTR) are the main performance measure for any alarm in the Alarms set. The central tendency of the measure and the variances are modeled incrementally to draw and reveal evolving understanding of system behavior.

The calculation of MTBE follows the same philosophy as MTBF. MTBE is defined as the arithmetic mean of the events from the elapsed time between inherent events (Alarm) triggered by the system as malfunction occurs during operation.

$$
\text { MTBE }=\sum \frac{\left(\text { STRAT }_{\text {DowNTIME }}-\text { Last }_{\text {Uptime }}\right)}{\text { number Of Fauilure }}
$$

Mean Time to Repair (MTTR) is a basic measure of the maintainability of repairable items. It represents the average time required to repair a failed component or device. Expressed mathematically, it is the total corrective maintenance time divided by the total number of corrective maintenance actions during a given period of time.

$$
\text { MTTR }=\sum \frac{(\text { RecoveryTime-EventTime })}{\text { numberOfFauilure }}
$$

MTBE and MTTR are estimated for the alarms triggered by the MO belonging to radio sites, each site will accumulate its estimation individually. Then the whole MTBE and MTTR of the RAN system are estimated. Some sites have many instantaneous MO classes, and in this case the MTBE is calculated for each individual MO and then averaged across the MO class. To realize such computation the log of previous event must be maintained or at least the last event information must be logged in the Alarm Frequency Matrix [19].

\section{RESULTS AND DISCUSSION}

Topology and Alarm data from a BSC of a typical MSP is collected from a study during a 2 week period in 2011 and parsed from alarm log plain text into tabular format. The BSC has 56 sites connected via 58 backhaul links. The adjacency list of the network was obtained, converted to an adjacency matrix and a corresponding directed graph is obtained, as shown in Fig. 2.

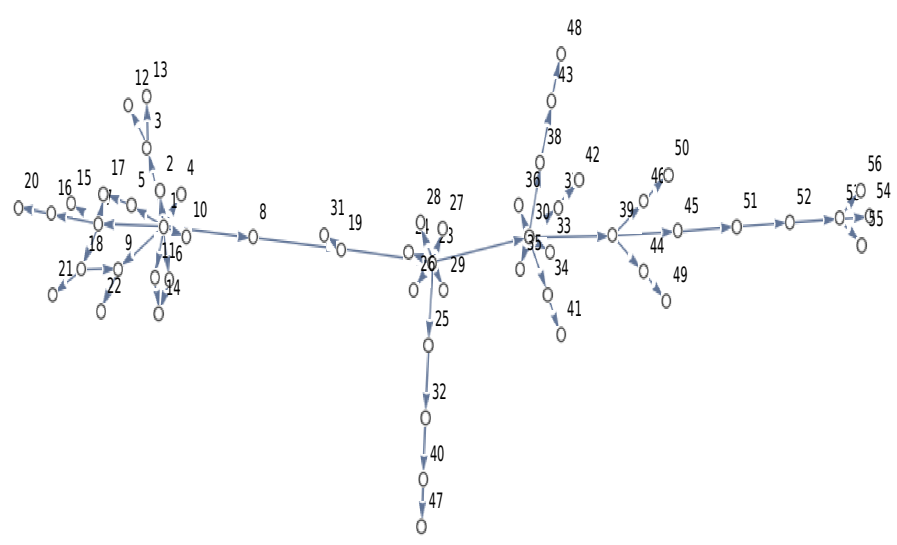

Fig. 2.Radio Sites Network Graph

Interestingly, the network graph is tree-like with root vertexes appearing with higher degrees. The average degree is found to be 2.07 with some sites have 4 or 5 times the degree (usually those are root vertexes) e.g. site 1 and 23 respectively. The degree vector of the sites is: $\{10,1,3,1,2,2,5,2,3,1,2$, $1,1,2,1,2,2,3,3,1,1,1,8,1,2,1,1,1,1,8,1,2,1,2,1,1$, $2,2,4,2,1,1,2,2,2,2,1,1,1,1,2,2,4,1,1,1\}$. The betweeness centrality is: $\{0,0,2,0,0.5,0.5,5.5,35,3,0,0.5$, $0,0,0,0,2,0,4,68,0,0,0,96,0,12,0,0,0,0,88,0,10,0,5$, $0,0,5,10,55,6,0,0,6,6,36,6,0,0,0,0,35,32,27,0,0,0\}$ and closeness centrality are found to be: $\{0.24,0,1,0,1,1$, $0.62,0.21,1,0,1,0,0,0,0,1,0,0.75,0.26,0,0,0,0.33,0,0.5$, $0,0,0,0,0.38,0,0.67,0,1,0,0,1,0.68,0.35,1,0,0,1,1$, $0.33,1,0,0,0,0,0.42,0.57,1,0,0,0\}$.

Betweeness results indicates the impact of nodes on the network and we can see that the relative importance is separated in two groups, sites with high betweeness (>27) and 
sites with low betweeness $(<27)$. This reflects directly the topology and has direct influence on the alarms related to transport issues. We note that the more central a node is the lower its total distances from all other nodes and the higher its closeness centrality (e.g. node 5).

Alarm text analysis and MOM analysis of 44155 alarms during a 2 weeks period from 30/09/20111 till 14/10/2011 results are shown in Fig.3. A total of 33 (out of 256 total alarms that could be generated by the BTS) were commonly observed for our analysis over 56 sites. Further, mapping alarm types to the service inventory reveals that the external fault, magazine address fault and internal fault are the 3 main contributors to service status impact across the BSC. Such outcome is an example of the power of the integrated RAN model presented in this paper, in quickly revealing service level information using topology information, MOM and alarm semantics extraction.

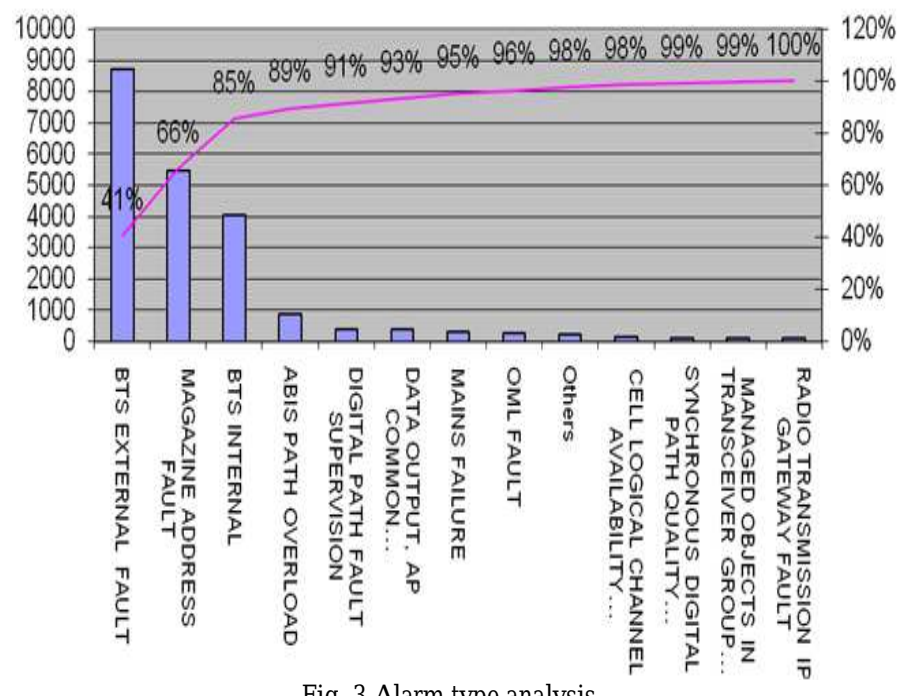

Fig. 3.Alarm type analysis

\section{CONCLUSIONS}

This paper presents a RAN model that integrates topology information, alarm events and resource information to provide a useful abstract representation of the network using MOM of the BTS. The MOM extracted from the alarm messaged and mapped in MOM tree topology that is then analysed resulting in improved fault diagnostic and interpretations. Results from a BSC in atypical MSPs showed that $85 \%$ of the faults within the BSC coverage area are attributed only to 3 alarm messages.

The insights gained from topology and network graph analysis are important in complementing alarm data mining and would be further explored using new data sets in future work. The RAN modelling framework presented in this paper provides a novel mapping mechanism of RAN events, faults and alarms and resources to service inventory. It provides a method for abstract level modelling of RAN resources while getting key insights from the RAN topology. This flexible handling of dynamic network events using MO class based model provides a robust fault diagnostic and direct mapping to service level. This forms part of a key challenge and provide necessary steps towards achieving autonomous network operation, service provisioning and service impact analysis to improve customer experience and business impact for MSPs. The above along with the analysis of performance comparisons from applying MTBE and MTTR measures on analysis of alarm text logs will be covered in future work relating to this paper.

\section{REFERENCES}

[1] ETSI, TS 132 111-1 - V4.0.0 - Universal Mobile Telecommunications System (UMTS); Telecommunication Management; Fault Management; Part 1: 3G fault management requirements (3GPP TS 32.111-1 version 4.0.0 Release 4), July 2011.

[2] Jakobson, G.; Weissman, M., "Alarm correlation," in Network, IEEE, vol.7, no.6, pp.52-59, Nov. 1993 doi: 10.1109/65.244794.

[3] Kettschau, H.-J.; Bruck, S.; Schefczik, P., "LUCAS - an expert system for intelligent fault management and alarm correlation," in Network Operations and Management Symposium, 2002. NOMS 2002. 2002 IEEE/IFIP, vol., no., pp.903-905, 2002 doi: 10.1109/NOMS.2002.1015639.

[4] ITU, Maintenance philosophy for telecommunication networks , M.20 (10/92),1992.

[5] Gardner, R.D.; Harle, D.A., "Methods and systems for alarm correlation," in Global Telecommunications Conference, 1996. GLOBECOM '96. 'Communications: The Key to Global Prosperity, vol.1, no., pp.136-140 vol.1, 18-22 Nov 1996 doi: 10.1109/GLOCOM.1996.594348

[6] Inokuma, H., "Event Log Analysis Service," in SICE-ICASE, 2006. International Joint Conference, vol., no., pp.5169-5172, 18-21 Oct. 2006 doi: 10.1109/SICE.2006.315678

[7] Jacques-H. Bellec and M-Tahar Kechadi. 2006. Towards a formal model for the network alarm correlation problem. In Proceedings of the 6th WSEAS International Conference on Simulation, Modelling and Optimization (SMO'06), Ana Maria Madureira (Ed.). World Scientific and Engineering Academy and Society (WSEAS), Stevens Point, Wisconsin, USA, 458463.

[8] Rish, I.; Brodie, M.; Odintsova, N.; Sheng Ma; Grabarnik, G., "Real-time problem determination in distributed systems using active probing," in Network Operations and Management Symposium, 2004. NOMS 2004. IEEE/IFIP , vol.1, no., pp.133146 Vol.1, 23-23 April 2004 doi: 10.1109/NOMS.2004.1317650

[9] Frohlich, P.; Nejdl, W.; Jobmann, K.; Wietgrefe, H., "Modelbased alarm correlation in cellular phone networks," in Modeling, Analysis, and Simulation of Computer and Telecommunication Systems, 1997. MASCOTS '97., Proceedings Fifth International Symposium on, vol., no., $\begin{array}{llll}\text { pp.197-204, Jan } & 1997\end{array}$ doi: 10.1109/MASCOT.1997.567613

[10] Venkat Venkatasubramanian, Raghunathan Rengaswamy, Surya $\mathrm{N}$ Kavuri, A review of process fault detection and diagnosis: Part II: Qualitative models and search strategies, Computers \& Chemical Engineering, Volume 27, Issue 3, 15 March 2003, 
Pages

313-326

ISSN

0098-1354,

http://dx.doi.org/10.1016/S0098-1354(02)00161-8.

[11] Cusick, J.J.; Ma, G., "Creating an ITIL inspired Incident Management approach: Roots, response, and results," in Network Operations and Management Symposium Workshops (NOMS Wksps), 2010 IEEE/IFIP , vol., no., pp.142148, 19-23 April 2010.

[12] Yong Tang; Xin Zhang, "Event Driving Based Alarm Correlation Approach by Using Petri Net," in Information Science and Engineering (ICISE), 2009 1st International Conference on , vol., no., pp.4865-4868, 26-28 Dec. 2009.

[13] Venkat Venkatasubramanian, Raghunathan Rengaswamy, Surya N. Kavuri, Kewen Yin, A review of process fault detection and diagnosis: Part III: Process history based methods, Computers \& Chemical Engineering, Volume 27, Issue 3, 15 March 2003, Pages 327-346, ISSN 0098-1354, Fhttp://dx.doi.org/10.1016/S0098-1354(02)00162-X.

[14] Roy Sterritt, Facing Fault Management as It Is, Aiming for What You Would Like It to Be. In: Soft-Ware 2002: Computing in an Imperfect World, Belfast, Northern Ireland. SpringerVerlag. $15 \mathrm{pp}$
[15] Hermann Wietgrefe and Klaus-dieter Tuchs and Klaus Jobmann and Guido Carls and Peter Fröhlich and Wolfgang Nejdl and Sebastian Steinfeld, "Using Neural Networks for Alarm Correlation in Cellular Phone Networks", In Proc. International Workshop on Applications of Neural Networks in Telecommunications, 1997.

[16] Matlab Tools for Network Analysis (2006-2011), MIT Strategic Engineering,http://strategic.mit.edu/downloads.php?page=matla b networks

[17] Michael Tiffany," Survey of Event Correlation Techniquesand Related Topics, http://www.tiffman.com/netman/netman.html, May 2002.Accessed 15/01/2016.

[18] Ma łgorzata Steinder, Adarshpal S. Sethi, A survey of fault localization techniques in computer networks, Science of Computer Programming, Volume 53, Issue 2, November 2004, Pages 165-194, ISSN 0167-6423, http://dx.doi.org/10.1016/j.scico.2004.01.010.

[19] Ekaette, E.U.; Far, B.H., "A framework for distributed fault management using intelligent software agents," in Electrical and Computer Engineering, 2003. IEEE CCECE 2003. Canadian Conference on , vol.2, no., pp.797-800 vol.2, 4-7 May 2003 